\title{
How Will the Results of the HEMO Study Impact Dialysis Practice?
}

\author{
Thomas A. Depner \\ Department of Medicine, Division of Nephrology, University of California, Davis, Davis, California
}

Uremia is the clinical consequence of poor renal function and is also the target of hemodialysis (HD) therapy. In the absence of a comprehensive understanding of uremia, the optimum therapy for an average patient with end-stage renal disease (ESRD) can be discovered by observing outcomes during a large clinical trial with randomly assigned dialysis options. The HEMO study was an ambitious effort to bypass traditional studies of disease pathogenesis (uremia) in favor of this type of population study (1). The motivation for embarking upon a population study like HEMO has been the persistently high HD mortality rate in the United States over the past 15 years (2).

Most clinical trials are tests of drug therapies where the primary goal is to determine whether or not the drug in question works, that is, whether treatment with the drug is better than treatment with a placebo. In contrast, the HEMO investigators were not hoping for a response, but had true equipoise, meaning that they were equally willing to accept a negative or positive response. In statistical terms, they were equally willing to accept or to reject the null hypothesis, which in this case was that the high dose of dialysis and/or high membrane flux would not have an effect. Potential benefits could be realized with either outcome, so "failure" of such a study only occurs when the outcome is equivocal, perhaps suggesting an effect but with no definite conclusion.

The HEMO study results were not equivocal, so the study can be viewed by all participants, patients, investigators, the dialysis industry, and the public in general as a resounding success. The study clearly demonstrated the futility of recent popular attempts to push the dose of dialysis beyond the Dialysis Outcomes Quality Initiative (DOQI) recommended minimum limit. Prior to the HEMO study, the minimum targeted dose, expressed as a single-pool $K t / V$ of 1.30 per dialysis treatment administered three times a week, was derived from a consensus of practicing nephrologists (3). Now, in addition to this learned opinion of nephrologists, the HEMO clinical trial provides objective evidence that the previously established minimum dose is also the optimum dose (4). In addition, the HEMO study shows us that improving the clearance of large molecules (inde-

Address correspondence to: Thomas A. Depner, MD, 4150V St., Sacramento, CA 95817, or email: tadepner@ucdavis.edu.

Seminars in Dialysis-Vol 16, No 1 (January-February) 2003 pp. 8-11 pendent of small molecules) by using high-flux membranes does not improve the survival of the average patient. Both of these observations have far-reaching implications with regard to the pathogenesis of uremia and its treatment.

\section{Applicability of the Study}

Therapeutic decisions based on data from the HEMO study should be made with an awareness of the type of patient included in the study, the outcomes that were monitored, and which outcomes were preselected (a priori) for study. Although several entry criteria were applied before patients were enrolled, these entry limits were primarily intended to improve the power of the study to distinguish an effect, using death as the primary outcome (5). Excluded patients were highly likely to die from causes other than inadequate dialysis (e.g., from cancer). The resulting randomized population had clinical characteristics that were representative of a cross section of patients currently dialyzed in the United States.

Nephrologists are sensitive to the quality as well as the quantity of life when deciding to begin, continue, or change dialysis treatments. Patient survival was the primary outcome criterion, but several clinically important secondary outcomes were also carefully monitored from the beginning of the HEMO study. First hospitalizations for cardiovascular events, first hospitalizations for infection, all nonaccess-related hospitalizations, and a $15 \%$ decline in serum albumin concentration from baseline were predesignated as secondary outcome monitors. For the first three secondary outcomes, allcause mortality was included to increase the power. In addition, prior to beginning the full-scale study, the principal investigators selected seven additional variables for scrutiny because they appeared to influence patient outcomes in previous studies. These variables were the patient's age, gender, race, diabetes as a cause of ESRD, number of years on dialysis when enrolled, baseline serum albumin, and an index of comorbidity. The importance of preselection and limiting the number of variables has been emphasized (1).

\section{Value of Clinical Trials}

Although expensive, clinical trials add a degree of certainty to treatment options that is not possible with 
other methods. Studies based on observation alone suffer from an inability to distinguish cause from effect. A recent example can be found in the correlation between cardiovascular disease and postmenopausal hormone replacement (6). The correlation between dialysis dose and survival seen in previous observational studies based on Medicare data or registries from other countries could have resulted from an inability to deliver higher doses to patients who are near death or from some other characteristic of patients at risk for death that also interfered with delivery of dialysis. By assigning patients randomly to treatment options in a clinical trial such as the HEMO study, the possibility of reverse causation was eliminated. Random assignments with an intentionto-treat analysis are key to avoiding misinterpretation of the data.

\section{HD Dose}

Separation of the dose groups took a major effort on the part of the individual dialysis center coordinators and the data coordinating center where statisticians carefully monitored the data and kept each coordinator informed about patients who strayed from their randomized assignments. In the end, the separation was especially good, with less than $5 \%$ overlap in the target treatment $e K t / V$ values (7). This means that the lack of response to the higher dose cannot be attributed to poor separation of the mean target values nor to inattentiveness on the part of the centers to keep each patient within his or her designated target throughout the study.

Nephrologists in general pay attention to the dialysis climate and respond quickly. Evidence of this can be found in the gradual increase in mean $K t / V$ that has occurred in the United States over the past 10 years. The average single-pool $K t / V$ has increased from approximately 1.10 in 1992 at the beginning of the HEMO study to 1.49 per dialysis in 2001 (2). Perhaps enthusiasm expressed by HEMO workers trying to recruit patients was misinterpreted by non-HEMO providers as a sign that the authorities supported increasing the dose to levels approaching the high goal of the HEMO study. The decision to publish individual clinic outcomes on the Internet, including the average dose of dialysis achieved in each dialysis clinic, probably contributed to this upward trend. During the early 1990s the mortality rate decreased, perhaps because of a reduction in the number of patients who were well below the threshold minimum value (8). But since 1995, despite a significant increase in average $K t / V$, no improvement in survival is evident even when the data are adjusted for age, diabetes, and other comorbid factors (2).

These observations, now supported by the HEMO study, indicate that for patients treated three times a week, a ceiling has been reached for the benefits expected by increasing the dose. The HEMO study also showed that this ceiling, the dose threshold above which improvements in mortality cannot be expected, is lower than some of the cross-sectional analyses had previously shown, and is possibly lower in men than in women (9).

\section{Concentration-Dependent Toxicity}

The question, "Why does giving more dialysis not help the patient?" requires a review of basic concepts. The concept that uremia, the target of dialysis, is a toxic state caused by accumulation of dialyzable solutes is fundamental. The severity of uremia must therefore correlate with the level of toxins in the body, which are presumably water soluble compounds found in the blood, the only tissue to which the dialyzer has access. For reasons that have been extensively reviewed and accepted (which I will not review here), nephrologists, including the HEMO investigators, have chosen the clearance of an easily dialyzable solute (urea) as the best measure of dialysis dose. However, even for continuous dialysis, clearance does not correlate linearly with (is not proportional to) solute levels. The concentration of creatinine, for example, is a hyperbolic function of native kidney clearance, a relationship well known to practicing nephrologists. Furthermore, the concentration of a toxin does not usually correlate linearly with its toxic effect, including death. Animal studies have shown that for known lethal toxins, this relationship is most often sigmoidal, with a threshold toxic level above which animals begin to die and a threshold lethal level above which all animals die (10). Applying these nonlinear relationships to intermittent treatments where rapidly changing concentrations reduce clearance efficiency, the curvilinear relationship between clearance and mortality is enhanced. When these concepts are combined and applied to the HEMO study, one can image how the dose/mortality curve reaches a plateau above which no further benefits can be extracted.

Recognizing the problem with urea disequilibrium, the HEMO investigators decided from the beginning to use $e K t / V$ instead of the traditional single-pool $K t / V$ as the adequacy index (7). Use of $e K t / V$ tended to straighten the relationship between mortality and dose, but only with respect to urea and only with regard to the relationship between patient clearance and dialyzer clearance. Other solutes maintain a curvilinear relationship between patient and dialyzer clearance and none of the other nonlinear relationships listed above were straightened by use of $e K t / V(7)$.

\section{Membrane Flux}

The other arm of the HEMO study was a random allocation of the same patients to treatment with either a standard-flux or a high-flux dialyzer. The flux distinction is actually a separation based on the clearance of high molecular weight (MW) solutes. Thus the HEMO study actually tested the effects of high and low clearances of high and low MW solutes. Of note, the clearance of high MW solutes can be completely separated from clearance of low MW solutes by selection of dialyzers. The dialyzers chosen may have had similar clearances of low MW solutes but markedly different clearances of $\beta_{2}$-microglobulin $\left(\beta_{2} \mathrm{M}\right)$, the marker for high $\mathrm{MW}$ solutes. Separation of clearances in the high MW range 
became a moving target to some extent as the study progressed because of reuse practices that increased or decreased the flux (11).

All dialysis membranes were either synthetic or made from cellulose derivatives that were relatively biocompatible, meaning that the effect of pure cellulosic complement-activating membranes was not tested. Although the latter type of dialyzer/membrane was in common use when the study started, the study investigators anticipated that use would drastically decline in later years, and this proved to be the case.

Absence of a significant reduction in mortality in the high-flux group can be translated to absence of an adverse effect of low-flux dialyzers. Trends in the secondary analyses, however, suggested a beneficial effect, and mortality appeared to be improved in patients with high vintage at baseline. High vintage patients were those maintained with HD for more than 3.7 years prior to randomizations. Conclusions about the latter await further analyses. The combined observations of borderline significant reductions in mortality and cardiovascular deaths in all patients, and significant improvement in survival in the high-vintage group should cause one to pause and consider high-flux membranes, especially today when the difference in cost of high-flux compared to standard membranes has narrowed.

\section{Subgroup Analysis}

Subgroup analyses are not yet complete, but there was a suggestion of a survival benefit at the higher dose in women and a suggestion of a benefit from high-flux dialysis in high vintage. For large subgroups, for example, men versus women or large racial groups, the outcome of the randomized groups cannot stray very far from the mean that showed no difference. The study was relatively large, however, and powered at $84 \%$ probability to detect a $25 \%$ reduction in mortality, so small differences can be significant. These subgroup reports will continue to appear and their significance will be debated with respect to clinical application and pathogenesis. The clinician should remember, however, that for the average patient neither mortality nor hospitalization rates, as defined by the four secondary outcome criteria, were improved by either the high-dose or highflux treatments. This means that large benefits from high dialyzer clearances of either large or small molecules in selected large subsets of patients cannot be expected when treatment is delivered in the present mode.

\section{Limitations of the Study}

As mentioned above, one must understand how the HEMO study was conducted before making decisions about individual patients. First, the patients were all dialyzed within a treatment time window of 2.5-4.5 hours. Because no discernible difference in outcome could be detected within these time constraints, it is unlikely that slight increases in treatment time would benefit the patient, but it is impossible to say whether giving much longer treatments (e.g., 8 hours) might confer a benefit. Second, the HEMO patients were all given three treatments per week, so recent reports of improved outcome in patients dialyzed more frequently are not inconsistent with the HEMO study results. As discussed above, the intermittent nature of $\mathrm{HD}$, as currently administered three times a week, is a major contributor to the curvilinear nature of the dose response, so increasing the frequency might be expected to help. Third, all hemodialyzers were reused a maximum of 20 times, so any adverse effect of reuse must be considered part of the outcome and cannot be distinguished from the dose effect.

\section{Patient Risks}

A strong note of thanks should go out to the patients who participated in the study. They put themselves at risk for the benefit of other patients. Although major efforts were made to avoid underdialysis, and in the end it appears that there was no risk, that information was not available to the patients during the study, so the decision to participate in each individual case was truly a sacrifice that was reflected in the consent forms signed by each patient. The decision by each individual patient and referring physician to participate in this study was made in the face of several public statements by otherwise well-meaning critics of the study advising against it because of the potential risk. These statements, published in nonscientific but widely distributed periodicals as late as January 2002, made it more difficult and expensive for the HEMO investigators to recruit patients. My hat is off to the patients who volunteered and accepted potential risks so that others might benefit. I also salute the more than 100 nephrologists who supported the study by allowing their patients to be randomized in the face of public criticism. Perhaps this study will serve as an example for potential critics of future studies to avoid the trap of being swayed by public opinion before definitive data are available.

\section{Conclusion}

The HEMO study conclusively answered the question of how much more dialysis should be given to patients based on the clearance of small or large molecules. The answer is that the current minimum standard for small molecule clearance per dialysis three times a week, factored for the patients volume of urea distribution, is enough and that giving more doesn't seem to help the average patient. In addition, use of more porous membranes to improve the clearance of larger molecules provides only minimal benefit for the average patient dialyzed three times a week. These results should not be construed as justifying a reduction in the dose of dialysis in all patients; continued adherence to the DOQI standard of 1.3 volumes per dialysis three times a week is recommended. Increasing the dose in women may 
provide a small benefit, but analysis of these data are not yet complete. Acknowledging that different individuals may have differing sensitivities to toxins and different rates of toxin accumulation, in clinical settings where specific symptoms or signs of uremia persist, the dose should be increased. Uncontrolled studies continue to suggest that increasing the frequency of dialysis may offer some benefit, so additional studies of frequency, a variable that was not addressed by the HEMO study, seem warranted.

What about the future? Will we see a decrease in the average dose as a result of this study? If a decrease in the average dose of dialysis occurs, it should not result from a wholesale decrease in all patients, but rather from a reduction in patients who are in the high range only. The ultimate effect would be a reduction in the spread of doses to a narrower dose window. Whether increasing the dose in certain subgroups will provide benefits can only be suggested by the HEMO study data and will require confirmation by other studies. Results of the HEMO study should not lead to complacency. Mortality remains high, especially in the United States, so dialysis providers must continue to look for ways to improve survival (i.e., to be dissatisfied with the practice of continuing to do what we do now).

\section{References}

1. Greene T, Beck GJ, Gassman JJ, Gotch FA, Kusek JW, Levey AS, Levin NW, Schulman G, Eknoyan G: Design and statistical issues of the hemodialysis (HEMO) study. Control Clin Trials 21:502-525, 2000

2. U.S. Renal Data System: USRDS 2002 Annual Data Report. Bethesda, MD: National Institutes of Health, National Institute of Diabetes and Digestive and Kidney Diseases, 2002

3. NKF-K/DOQI Clinical practice guidelines for hemodialysis adequacy: update 2000. Am J Kidney Dis 37(suppl 1):S7-S64, 2001

4. Hakim RM, Depner TA, Parker TF: In depth review: adequacy of hemodialysis. Am J Kidney Dis 20:107-123, 1992

5. Eknoyan G, Levey AS, Beck GJ, Agodoa LY, Daugirdas JT, Kusek JW, Levin NW, Schulman G: The hemodialysis (HEMO) study: rationale for selection of interventions. Semin Dial 9:24-33, 1996

6. Risks and benefits of estrogen plus progestin in healthy postmenopausal women: principal results from the Women's Health Initiative randomized controlled trial. JAMA 288:321-333, 2002

7. Depner T, Beck G, Daugirdas J, Kusek J, Eknoyan G: Lessons from the hemodialysis (HEMO) study: an improved measure of the actual hemodialysis dose. Am J Kidney Dis 33:142-149, 1999

8. Gotch FA, Levin NW, Port FK, Wolfe RA, Uehlinger DE: Clinical outcome relative to the dose of dialysis is not what you think: the fallacy of the mean. Am J Kidney Dis 30:1-15, 1997

9. Eknoyan G, Beck GJ, Cheung AK, Daugirdas JT, Greene T, Kusek JW, Allon M, Bailey J, Delmez JA, Depner TA, Dwyer JT, Levey AS, Levin NW, Milford E, Ornt DB, Rocco MV, Schulman G, Schwab SJ, Teehan BP, Toto $\mathrm{R}$ : Effect of dialysis dose and membrane flux on mortality and morbidity in chronic hemodialysis patients: primary results of the HEMO study. $N$ Engl J Med : (in press)

10. Gibaldi M, Perrier D: Pharmacokinetics. New York: Marcel Dekker, 1982

11. Kaplan AA, Graeber CW: Dialysate protein losses with bleach processed polysolphone dialyzers. Kidney Int 47:573-578, 1995

\section{Frank A. Gotch}

Department of Medicine, University of California, San Francisco, School of Medicine, San Francisco, California

This brief commentary reviews the HEMO study outcome results from the perspective of the dialysis dosing controversy over the 20 years since the National Cooperative Dialysis Study (NCDS) was completed in 1981. It is important to note, however, that the HEMO study has not yet been formally published and thus the following comments are based only on the presentation of main study results by Gary Eknoyan at the spring National Kidney Foundation (NKF) meeting in Chicago earlier this year (1).

The NCDS was a landmark study (2) and, prior to HEMO, the only study of clinical outcome in hemodialysis (HD) correlated to prospectively randomized dialysis doses. In retrospect it was a tiny study of only 160 patients observed a minimum of 6 months and distributed in four treatment arms, but it was a powerful study because it was prospectively randomized and the randomized doses were distributed over a very wide range. Outcome in the NCDS was reported as the probability of failure $(\mathrm{PF})$, which was a global clinical

Address correspondence to: Frank A. Gotch, MD, 144 Belgrave Ave., San Francisco, CA 94117, or email: fgotchsf@cs.com.

Seminars in Dialysis-Vol 16, No 1 (January-February) 2003 pp. 11-13 composite of de novo uremic symptoms, hospitalizations, and death occurring during follow-up.

Analysis of clinical outcome in the NCDS as a function of $s p K t / V$ (3) is shown in Fig. 1, where the probability of failure is normalized to the value at $s p K t /$ $V=1.45$ and expressed as relative probability of failure (RPF). The 10 groups with 16 patients in each group are mapped to RPF and $s p K t / V$ coordinates in Fig. 1, where the wide dispersion of both RPF (5.8 to 0.5) and $s p K t / V$ ( 0.42 to 1.45$)$ in the NCDS can be seen. Three highly significant regressions of RPF on $s p K t / V$ were reported (3) consisting of discontinuous, exponential, and linear functions. Although it was not mathematically possible to clearly show one relationship was superior to the others, we felt the data conformed best to the discontinuous function depicted in Fig. 1, which resulted in the conclusion that optimal therapy was achieved with $s p K t /$ $V \geq 1.0$, a value $25 \%$ higher than the apparent breakpoint at $s p K t / V=0.8$. However, if RPF in the NCDS were more correctly described by the exponential regression shown in Fig. 1, a very significant decrease in RPF would be predicted for $s p K t / V>1.0$. This exponential curve would suggest a $70 \%$ decrease in RPF if $s p K t / V$ were increased from 1.0 to 1.75 .

Over the subsequent 20 years a number of observational studies have been reported suggesting the optimal $s p K t / V$ is in the range $1.4-1.8$ or even greater. Some of 
these studies correlated mortality to mean values of $s p K t /$ $V$ distributed over a wide range in the patient population $(4,5)$, calling into question the validity of using the mean value (6). A very recent large observational study based on dose estimates derived from the urea reduction ratio (URR) suggests a linear decrease in mortality over all doses observed up to an estimated $s p K t / V$ of 1.8 (7).

As noted above, the HEMO study was prospectively randomized and had 10 times as many patients as the NCDS, with observations extending over up to 6 years of follow-up compared to 6 months in the NCDS. Dr. Eknoyan reported mean doses of $s p K t / V$ of 1.32 and 1.71 (URR 66\% and 75\%) in the standard and high-goal treatment arms, respectively. The details will undoubtedly be described much more fully in peer reviewed publications by HEMO investigators. The higher doses of dialysis did not improve survival, reduce hospitalizations, or maintain serum albumin. However, Dr. Eknoyan reported that in women the higher dose may have been associated with improved survival, and further analyses of this and other subgroups are under way.

The preliminary results for the HEMO study and the very recent analysis of Health Care Financing Administration (HCFA) observational data (7) are shown graphically in Fig. 2 and are compared to the exponential regression for NCDS data in Fig. 1. The HEMO data are arbitrarily plotted for the standard and high-goal arms at a relative risk of mortality (RRM) of 1.0, since there was no change in mortality over the dose range studied. The HCFA data (where dose was measured from URR) are also plotted in Fig. 2 and indicate that RRM decreased substantially as estimated $s p K t / V$ increased over the range 1.0-1.7. It can be seen in Fig. 2 that outcomes in the HEMO and HCFA studies are correlated to doses well distributed over the controversial segments of the discontinuous and exponential outcome curves for the NCDS data in Fig. 1 and might be expected to resolve the 20-year controversy.

These two studies are further compared in Fig. 3, where RRM as a function of both URR and $s p K t / V$ are shown. The striking differences between the two studies are best illustrated here. In the HEMO study, when dose was prospectively increased from $s p K t / V 1.31$ to 1.75 with a corresponding URR increase from $66 \%$ to $75 \%$, there were no improvements in any clinical outcome

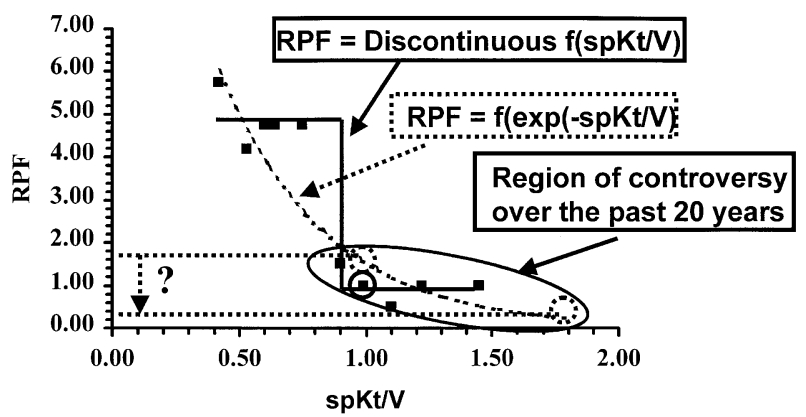

FIG. 1. The relative probability of failure (RPF) in the NCDS (1985). The outcome data were interpreted to conform best to a discontinuous function of $s p k t / V$; however, an exponential fit was also reported. Over the past 20 years these two interpretations have been highly controversial over the range $1.0 \leq s p K t / V k<1.7$.

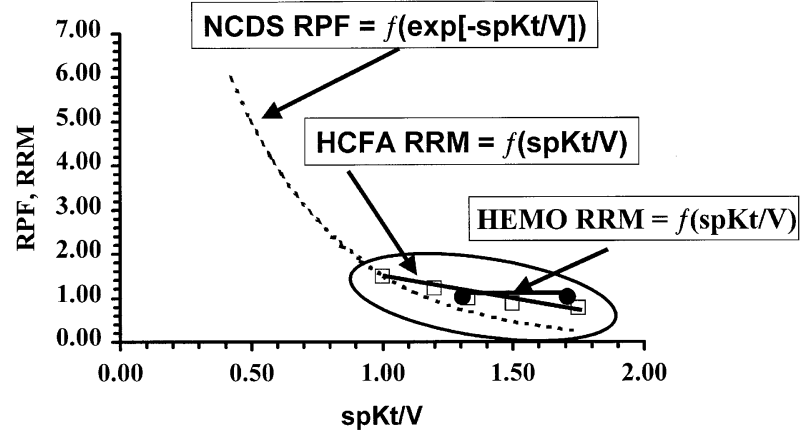

FIG. 2. RRM in the HEMO study and in a recent HCFA study compared to the HEMO and HCFA studies are targeted to the controversial region of $1.0 \leq s p K t / V \leq 1.7$. See the text for a discussion of these studies.
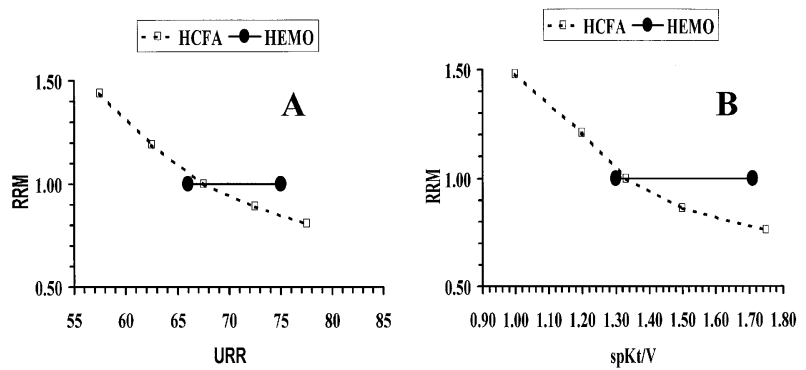

FIG. 3. Comparison of RRM as $f(\mathrm{URR})$ and $f(s p K t / V)$ in HCFA and HEMO studies. See the text for a discussion.

measures as noted above. Further, these two levels of dosing were held constant for up to 6 years of follow-up. In contrast, the HCFA data analysis over 1 year of follow-up (but with many more patients and deaths observed) showed a linear decrease in observed RRM from 1.0 to 0.76 as observed URR increased from $67.5 \%$ to $77.5 \%$. These observations led the authors to conclude that adequate dialysis appears to require a URR > 75\% and $s p K t / V>1.75$. What can account for such strikingly different results between the prospectively randomized 6-year HEMO trial and the 1-year observational analysis of HCFA data?

One partial explanation for the difference may lie in the relative reliability of dose measurement. The delivered dose in the HEMO study was monitored monthly with before and after blood urea nitrogen (BUN) levels subjected to formal kinetic analysis to calculate the delivered dose and change the prescription as needed to maintain the targeted doses. In this way the frequency of spurious URRs is reduced to near zero and URR corresponds very closely with delivered $s p K t / V$ in each individual patient. In contrast, the HCFA analysis is based only on URR values reported to the nearest $5 \%$ and there is no check on the validity of data. The most common error in URR is almost certainly overestimation due to recirculation late in dialysis combined with faulty technique in drawing the postdialysis sample. Such process errors could be enhanced in some dialysis facilities under economic pressure and striving for maximal URRs to report to the HCFA, and result in systematic overestimation of URR, particularly in the higher ranges. 
It is impossible to know how far the HCFA curves might be displaced to the right in Fig. 3 due to some systematic overestimation of URR values. URRs in the range of 72.5-77.5\% represent very high doses of dialysis and, in the absence of kinetic validation of the delivered dose, must be questioned. In the medium and large patient groups, URRs of $72.5-77.5 \%$ were observed in $56 \%$ and $47 \%$ of patients, respectively, and correspond roughly with $s p K t / V$ values of 1.6-1.8. Assuming an average volume of $35 \mathrm{~L}$ for the medium-sized patients and treatment time of 210 minutes, the dialyzer whole blood clearances range from 310 to $355 \mathrm{ml} / \mathrm{min}$. At an average $Q b$ of $450 \mathrm{ml} / \mathrm{min}$, these clearances would require dialyzer whole blood Ko As of 900-1400 ml/min, higher than most dialyzers readily available. Even higher clearances or much longer treatment times would be required for large patients, with $47 \%$ of URRs in the range $72.5-77.5 \%$. These considerations raise questions about the validity of the high URR values in HCFA data that were not subjected to kinetic validation of the dose. In this regard it is also important to emphasize that the HEMO study provides the first data comparing clinical outcome determined as a function of randomized doses accurately measured by kinetic modeling to outcome correlated to observed approximate measures of dose such as URR.

Another and perhaps more important reason for the difference in the two studies is inherent in the nature of randomized and observational studies. There may well be unmeasured factors in the HCFA dataset that influenced both URR and mortality that the investigators were unable to control for in the analysis. This type of bias is unlikely in the HEMO study because patients were randomized into the two $K t / V$ groups.

Is it possible that the HEMO study was not adequately powered to rule out the magnitude of mortality decrease observed in the HCFA data? Over the range $1.33 \leq$ spKt/ $V \leq 1.75$ in the HCFA data, the RRM fell from 1.00 to 0.76 , or $24 \%$. The RRM for the $K t / V$ intervention in HEMO was $0.96(p=0.52)$ with $95 \%$ confidence limits of $0.84-1.09$ (16\% reduction to $9 \%$ increase in RRM). Thus the maximal possible benefit compatible with the HEMO study results would be a $16 \%$ reduction in RRM, which is inconsistent with the $24 \%$ reduction observed in the HCFA data. The 95\% confidence limits on the four main secondary clinical composite outcomes reported by Eknoyan (1) were very tight, with upper bounds for the relative risk reduction of the high $K t / V$ goal ranging from 9 to $13 \%$, and provide further strong evidence in support of the null result in the HEMO study.

Although we must await full publication of the HEMO study and subgroup analyses to reach final conclusions, the outcome results reported to date are very convincing that a kinetically measured $s p K t / V$ of 1.3 and corresponding URR of $65 \%$ define the average fully adequate dose of thrice weekly HD. However, in individual patients with a range of treatment times and ultrafiltration, the best definition of an adequate dose would be an $e K t / V$ of 1.2 , the rounded up value that was actually modeled and achieved in the standard arm. Both $s p K t / V$ and URR may vary substantially in individual patients relative to $e K t / V$ due to wide ranges of treatment times and ultrafiltration. It should also be noted that the average treatment times were 3.2 and 3.7 hours in the standard and high goal arms and the shorter treatment time had no adverse effect on outcome. Further analyses of other observational data in conjunction with randomized HEMO data may help to resolve the large differences between the randomized and observational studies that still persist.

\section{References}

1. Eknoyan G: The hemodialysis (HEMO) study, an NIH-NIDDK sponsored randomized, multicenter clinical trial. Paper presented at the NKF Spring Clinical Nephrology Meeting, Chicago, IL, April 2002

2. Lowrie E, Lard N (eds): Cooperative dialysis study. Kidney Int 23(suppl 13):S1-S122, 1983

3. Gotch F, Sargent J: A mechanistic analysis of the National Cooperative Dialysis Study (NCDS). Kidney Int 28:526-534, 1985

4. Hakim R, Bryer J, Ismail N, Schullman G: Effects of dose of dialysis on morbidity and mortality. Am J Kidney Dis 23:661-669, 1994

5. Parker T, Husni L, Huang W, Lew N, Lowrie E: Survival of hemodialysis patients in the United States is improved with greater quantity of dialysis. Am J Kidney Dis 23:670-680, 1994

6. Gotch F, Levin N, Port F, Wolfe R, Uehlinger D: Clinical outcome relative to the dose of dialysis is not what you think: the fallacy of the mean. Am J Kidney Dis 30:1-15, 1997

7. Port F, Ashby V, Dhingra R, Roys E, Wolfe R: Dialysis dose and body mass index are strongly associated with survival in hemodialysis patients. $J$ Am Soc Nephrol 13:1061-1067, 2002

\section{Friedrich K. Port* and Robert A. Wolfe}

*University Renal Research and Education Association (URREA), Ann Arbor, Michigan, and Department of Biostatistics, Kidney Epidemiology and Cost Center, University of Michigan, Ann Arbor, Michigan

An oral report on the HEMO study at the 2002 spring meetings of the National Kidney Foundation (NKF) indicated no difference in mortality for patients randomized to two dialysis dose groups of $e K t / V=1.05$

Address correspondence to: Friedrich K. Port, MD, MS, URREA, 315 West Huron, Suite 260, Ann Arbor, Ml 48103, or email: fport@urrea.org.

Seminars in Dialysis-Vol 16, No 1 (January-February) 2003 pp. 13-16 versus 1.45 . This result has been a surprise to many in the renal community, including the expert panel of the Dialysis Outcomes Quality Initiative (DOQI) guidelines (1) whose information was based on several observational studies.

Randomized controlled clinical trials have several design characteristics that avoid the biases that can occur in observational studies. However, more details are needed from the HEMO study before appropriate conclusions can be reached about the following issues. 
What are the results? To date, the conclusions have been stated as a failure to reject the null hypothesis of no difference in outcomes for the two treatment groups or that the outcomes were similar for both dialysis dose study groups. However, standard textbooks indicate that, "When the test statistic falls in the acceptance region, we will not reject, rather than accept, the null hypothesis. Further conclusions may be made by calculating an interval estimate for the parameter..." (2). Thus such negative results should not be interpreted as proof that there is no difference, but should instead be interpreted based on a confidence interval range of plausible differences in outcomes, which account for the uncertainty in the data from the trial. The confidence interval will clarify the size of the potential beneficial effect in the higher-dose group.

How general are the results? Are there differences in outcomes among subgroups of patients that preclude a broad conclusion about all patients, as suggested in the preliminary reports? Are there different means of achieving a certain dose level that might be more effective? The HEMO study does not offer definitive conclusions about the efficacy (or lack of it) of the achieved difference in $K t / V$, but instead yields an evaluation only of the specific protocols used to achieve the differences in dialysis dose.

Randomized clinical trials have been rare in dialysis. The last major trial in the United States, the National Cooperative Dialysis Study (NCDS), more than 20 years ago, suggested that longer treatment time was associated with better outcomes. However, since this finding had a $p$-value of 0.056 , it was considered not significant and therefore not relevant (3). Subsequent analysis of the same data by Gotch and Sargent (4) led to the concept of $K t / V_{\text {urea }}$ as an indicator of dialysis dose for small molecules and as a predictor of outcomes.

Numerous observational studies have since confirmed that low dialysis dose is associated with higher mortality. The DOQI guidelines, based on available evidence, recommended a minimum single-pool $K t / V$ of 1.2 , which corresponds to a double-pool or equilibrated $e K t / V$ of approximately 1.05 (1). Unresolved issues included whether the dose should be substantially higher than suggested by the DOQI and whether delivering the same dose in a longer treatment time $(t)$ would offer further advantages to patients. Port et al. (5) showed that patients treated with a URR $>75 \%$ had a lower mortality risk than patients treated with a URR of $70-75 \%$, on average. The $70-75 \%$ URR level is substantially above the recommended URR of greater than $65 \%$. Given the current clinical practice of offering 3- to 4-hour dialysis on a thrice-weekly schedule, the effect of varying treatment time is difficult to assess. However, single-facility studies and the Dialysis Outcomes and Practice Patterns Study (DOPPS) provide suggestive evidence that delivering the same dose in sessions longer than 4 hours is associated with lower mortality risk (6). However, given the small sample size for these studies, the $p$-value was greater than 0.10 .

Thus evidence from several observational studies suggested that for thrice-weekly hemodialysis (HD), a greater dialysis dose is better. These findings have been further corroborated with several reports on experience after switching from standard to daily HD. Most reports showed clear clinical advantages after the change to more frequent dialysis, whether it was delivered as long nightly dialysis or short daytime dialysis (7). However, large randomized trials regarding the frequency and duration of dialysis are lacking.

The long-awaited results from the HEMO study failed to confirm these findings from prior observational studies overall. This new evidence may be confusing to practicing clinicians. Randomized trials are usually considered the "gold standard," even when not blinded, as was the case in the HEMO study.

There are important differences between observational studies and randomized clinical trials (8). For example, ethical reasons may prevent a trial to evaluate low-dose $\mathrm{HD}$, while observational studies can cover a wide range of practices, including low doses. Cost issues demand that clinical trials limit the sample size to a minimum according to power calculations. Although observational studies suffer less from these limitations, their findings of correlations are limited by confounding and do not allow for conclusions of causality. For example, if higher dialysis doses are given to healthier patients, lack of adjustment for the patient's health status would not permit differentiation of patient selection from a beneficial effect of a higher dose. Therefore observational studies need to strive to adjust for numerous potential explanatory variables.

Randomized trials overcome the problem of confounding because the random assignment should make all variables, except the one or two study factors, similar in both groups. Occasionally some variables turn out to be different by chance and, if recognized, statistical adjustment can correct for such differences. Generalizability of the results depends on the exclusion of certain patient groups, such as patients with high body weight in the HEMO study. Large population-based observational studies are more likely to yield conclusions applicable to the entire population.

With this background we can evaluate the currently available evidence from the HEMO study. The primary analysis showed no significant difference in outcomes for the two dialysis-dose groups. This analysis used the intention-to-treat approach according to the original assignment. It will be important to know if the intended dose separation for the two groups was actually achieved for all patients. Should there be a substantial overlap, the negative results could be partially explained by lack of power and misclassification of the dose groups. Secondary analyses to evaluate the actually delivered dose and correlate it with the outcomes can help to distinguish between a failure to achieve desired protocols versus the lack of an outcome difference between the protocols. Yet such secondary analyses will reduce the benefit of randomization, making "all else equal."

Patient selection for the HEMO study required that all study patients could achieve the higher dose level of $e K t /$ $V=1.45$ before randomization. This requirement excluded larger-sized patients so that body weights were almost always less than $90 \mathrm{~kg}$. This exclusion deserves further exploration because of the ongoing debate about 
a J-shaped correlation of mortality risk by dialysis dose. Body mass index (BMI) may serve as a proxy for nutritional status. Based on data from Wolfe et al. (9), BMI is correlated with body weight (likely because in large populations weight varies more than height). Thus the J-shape of an increase in risk at the highest dose levels is largely explained by the confounding by body size, in that smaller patients tend to receive a higher dose than larger patients (9). Smaller patients are potentially undernourished, which is clearly associated with higher mortality (10). Recent data by BMI tertile suggest that the exclusion of larger patients would not reduce the expected association with mortality (5) and therefore should not explain the negative finding in the HEMO study.

Observational studies can be confounded by factors that may explain the observed results. Therefore it is critical for such studies to have a large sample size and to adjust for numerous factors. Many of the studies (9) adjusted for demographic factors, a large number of comorbidities, and some laboratory values and showed that a low dialysis dose was associated with higher mortality risk. We should look for potential explanations for the findings in the reports from observational studies.

Bias would arise if patients receiving a higher dose were healthier to begin with than were those receiving lower doses. Studies by Wolfe et al. (9) adjusted for comorbid conditions at the beginning of the study. The most recent study by Port et al. (5) adjusted for comorbidities observed at an earlier time, yet the results were consistent with a cross-sectional study that adjusted for numerous comorbidities at the beginning of the study. A more refined adjustment for severity of comorbidity would reduce the potential for such bias, but such adjustments are seldom perfect.

The measurement of dialysis dose is likely to be less accurate in observational studies than in the HEMO study, but this should have biased the observational results to the null (i.e., to finding no difference) rather than to finding significant and large differences in mortality risk by dialysis dose. Similarly, observational studies evaluated the baseline dose of dialysis, which could change during follow-up, while the HEMO study tried to maintain the delivered dose during follow-up.

Bias would arise in observational studies if better doctors and teams deliver higher doses of $e K t / V$. Thus better medical care rather than higher dose could have led to the observed correlation. A recent study tried to consider and minimize the role of other practice patterns: Wolfe et al. (11) studied practices and outcomes at the dialysis facility level rather than at the patient level. We found that the adjusted mortality based on standardized mortality ratio was higher in dialysis units that had a larger fraction of their patients receiving suboptimal URR of less than $65 \%$ or having a hematocrit of less than $33 \%$ or using a central venous catheter. When adjusting for these three practice factors simultaneously, dialysis units with more patients receiving a low dialysis dose still had a higher standardized mortality. Thus when the practice of anemia and vascular access care was similar, dialysis units that had a larger fraction of patients receiving a low dialysis dose had higher mortality.
In randomized clinical trials it is also possible that other factors modified the results. Specifically, in the unblinded study environment, other unmeasured factors may have been given extra attention in the high-dose group, which could reduce the expected difference in outcomes and thus lead to a bias in the randomized trial.

The role of residual renal function needs to be considered. There is evidence that residual renal function plays an important role and that clearance of molecules larger than urea is beneficial. Therefore it does not seem appropriate to consider native renal function as equivalent to dialysis clearance. Observational studies have tried to overcome this problem by excluding to a large extent patients with residual renal function-for example, by starting the study after 15 months of dialysis therapy (5). Observational studies suggest that higher doses of dialysis are associated with lower mortality after 1 year of dialysis (after residual renal function has declined), with only small effects during the first year of dialysis. Presence of residual renal function in a study group would limit generalization of the results to patients with little or no residual function.

\section{Integration of the Discrepant Findings for Clinical Practice}

The low target dose for the HEMO study was $e K t / V=1.05$, which corresponds approximately to a single-pool $K t / V$ of 1.2 and a URR of $65 \%$. The target in a trial must not be confused with a mean value or goal in clinical practice because the spread around the mean is much wider in clinical practice than in a controlled study environment. Therefore this study must not be interpreted to suggest that dialysis units reduce their target dialysis dose to an $e K t / V$ of 1.05 . The target must be substantially higher to ensure that all patients receive an $e K t / V$ of at least 1.05. Data for a lower $e K t / V$ are only available from observational studies and they are very consistent in showing an association with higher morbidity and mortality. Since even the high dialysis dose of the HEMO study $(e K t / V=1.45)$ is still indicative of an averaged creatinine clearance of less than $20 \mathrm{ml} / \mathrm{min}$ and since daily HD is of benefit to patients, it seems to be advisable not to reduce dialysis dose at present.

As more details of the HEMO study results become available it will be interesting to learn the width of the confidence interval for the mortality risk at the higherdose level. It is possible that the observed risk for the higher dose group in the HEMO study is consistent (i.e., overlapping) with the prior observation of a reduced mortality risk. While the findings are reported as consistent with no benefit from the higher dose, this information may also be consistent with prior observations of a benefit.

The HEMO Study results were presented at the American Society of Nephrology meeting in October 2002. The mortality risk for the high dose group (mean $e K t / V=1.53$ ) versus the lower dose group (eKt) $V=1.16)$ was $\mathrm{RR}=0.96$ and the $95 \%$ confidence interval for this not-significant finding was $0.84-1.10$. For this wide spread of the two dose groups, the more 
recent observational studies would have estimated the mortality RR to be 0.86 . Since this value is within the confidence interval of the HEMO Study, one can conclude that the results of the HEMO Study agree with both the null hypothesis (that higher does has no effect) and the prior hypothesis (observational studies that show a significant benefit to higher dose). Thus more study is needed, including further exploration of the HEMO Study. At this time we caution against a dose reduction, as did some HEMO Study investigators during the discussion of their results.

\section{References}

1. National Kidney Foundation Dialysis Outcomes Quality Initiative (NKFDOQI) Clinical practice guidelines for hemodialysis adequacy. Am J Kidney Dis 30(suppl 2):S15-S66, 1997

2. Mendenhall W, Beaver RJ (eds): Introduction to Probability and Statistics, 9 th ed. Belmont, CA: Duxbury Press, 1994
3. Lowrie EG, Laird NM, Parker TF, Sargent JA: Effect of dialysis prescription on patient morbidity. $N$ Engl J Med 305:1176-1180, 1981

4. Gotch FA, Sargent JA: A mechanistic analysis of the National Cooperative Dialysis Study (NCDS). Kidney Int 28:526-534, 1985

5. Port FK, Ashby VB, Dhingra RK, Roys E, Wolfe RA: Dialysis dose and body mass index are strongly associated with survival in hemodialysis patients. J Am Soc Nephrol 13:1061-1066, 2002

6. Held PJ: Dialysis dose and treatment time in the DOPPS. Paper presented at the International Dialysis Conference, Phoenix, AZ, 2002

7. Woods JD, Port FK, Orzol S, Buoncristiani U, Young E, Wolfe RA, Held PJ: Clinical and biochemical correlates of starting "daily" hemodialysis. Kidney Int 55:2467-2476, 1999

8. Port FK: The role of observational studies as compared to clinical trials in ESRD research. Kidney Int 57(supp1 74):S3-S6, 2000

9. Wolfe RA, Ashby VB, Daugirdas JT, Agodoa LY, Jones CA, Port FK: Body size, dose of hemodialysis and mortality: results from USRDS Special Study. Am J Kidney Dis 35:80-88, 2000

10. Leavey SF, Strawderman RL, Jones CA, Port FK, Held PJ: Simple nutritional indicators as independent predictors of mortality in hemodialysis patients. Am J Kidney Dis 31:997-1006, 1998

11. Wolfe RA, Hulbert-Shearon TE, Roys EC, Port FK: Standardized mortality (SMR) and hospitalization (SHR) ratios by dialysis unit practice pattern of anemia adjusted for dialysis dose and vascular access type. J Am Soc Nephrol 11:A1299, 2000

\section{Robert M. Lindsay and Peter G. Blake}

Optimal Dialysis Research Unit and the London Health Sciences Center, London, Ontario, Canada

The presentation and publication of the results of the HEMO study is a landmark event in the history of hemodialysis (HD). This study is methodologically sound and the investigators who carried it out are to be congratulated. The results of the study have, however, been surprising and even disturbing to many nephrologists. The notion that higher doses of HD lead to better patient outcomes had become widespread. Now there is a need to pause and reconsider. This, however, is a good process for our discipline to undergo. It leads us to question our preconceived notions and it inspires us to look for new ways of delivering better treatment.

How should this study influence our practice? This question can be considered on two levels. First, there is the question of how it should alter prescription of conventional three times a week HD; second, is the broader issue of whether alternative HD regimens may be required to improve outcomes substantially. This review addresses these two topics separately.

\section{Prescription of Conventional HD}

In conventional HD, the HEMO study tells us that targeting a single-pool $\mathrm{Kt} / \mathrm{V}$ of $1.25 /$ treatment gives outcomes just as good as targeting a higher value of 1.65. Obviously all patients in our HD units should be receiving at least the lower value of $1.25 /$ treatment,

Address correspondence to: Robert M. Lindsay, MD, Optimal Dialysis Research Unit and the London Health Sciences Center, 800 Commissioners Rd, London, Ontario, NOA4G5 Canada.

Seminars in Dialysis-Vol 16, No 1 (January-February) 2003 pp. 16-19 corresponding to a urea reduction of $65 \%$. An important question that arises, however, is how we should deal with the significant minority of patients who are already receiving $K t / V \mathrm{~s} \geq 1.4 /$ treatment. These patients are typically those of below average body size with dialysis times of 3.5-4.5 hours. Should we reduce their dialysis times on the theory that outcomes will not be adversely affected? After all, most patients do not wish to spend any more time on HD than is required to maintain their health. Such an approach would therefore seem justified.

However, there are important caveats to consider before treatment times are reduced. The HEMO study did not directly examine the effect of time on patient outcomes. Randomization was by clearance and flux only. Inevitably, of course, the high- $K t / V$ group did receive more dialysis time, but it is of interest that the difference was only 30 minutes. Thus the standard group had a mean dialysis time of 3 hours, while the high- $K t / V$ group had a mean of 3.5 hours. The surprisingly short times required to achieve the higher $K t / V$ reflect the relatively low body weight of patients who were eligible for the HEMO study.

Therefore the study does not allow us to comment on whether dialysis times of 4 hours or longer, as are used in many countries, are superior to dialysis times of 3 hours. Two decades ago the National Cooperative Dialysis Study (NCDS) (1) addressed this issue. At that time the investigators found, in a statistically very underpowered study, that patients with longer treatment times (i.e., 4-5 hours) had borderline statistically significant better outcomes than patients with shorter treatment times.

The concern in all this, obviously, is that dialysis time is a surrogate for control of extracellular fluid volume and thus blood pressure, and that this in turn may be critical for cardiovascular outcomes. There is little doubt that the contemporary HD population has markedly higher 
rates of cardiovascular disease and hypertension than was the case at the time of the NCDS. There is therefore a significant concern that reductions in dialysis time may adversely affect volume status, hypertension, and ultimately patient outcomes. Notwithstanding all this, a reasonable compromise might be as follows. Patients who have a $K t / V$ in excess of $1.4 /$ treatment could have their dialysis time cautiously reduced if their blood pressure is very well controlled and if their weight gains are modest. In this regard, good blood pressure control is defined as readings consistently less than $135 / 85 \mathrm{mmHg}$. Patients who do not fulfill these criteria risk having their volume status and blood pressure adversely affected if times are reduced.

New HD patients should be modeled to achieve a $K t / V$ of $1.3 /$ treatment, but the required time to reach this target may not be sufficient to deal with fluid gains and optimize volume status and blood pressure. The crucial point here is that dialysis time should not be determined solely by the need to achieve a given clearance target. Rather it should also be strongly influenced by the need to optimize volume status and blood pressure control. Of course, it is recognized that in many patients dialysis treatment times of 4-5 hours are still not associated with optimal blood pressure control. This highlights the need to look at new paradigms for HD.

\section{The Prescription for the Future?}

The current treatment paradigm for HD of three treatments per week each lasting 3-4 hours has been followed almost worldwide for the past 25 years or more. This treatment prescription is not based on optimizing physiology, but rather was developed as a compromise between patient acceptance, what we knew about adequacy of dialysis, and economic considerations, with these three often moving in different directions. Patient acceptance of HD treatment is marginal at best; the treatment being plagued with intradialytic and postdialytic symptoms, thus the patient often begs for a shorter treatment. In some areas of the world, the economics have also led to shorter treatments and adequacy might be compromised. Such a compromise in turn may have an adverse effect on population morbidity and mortality statistics.

Even with the confusion following the HEMO study, there must be at least a threshold for a delivered singlepool $K t / V$ (perhaps 1.25/treatment?) below which results in adverse outcome. There are many different ways to influence the delivered dose of dialysis, for example, by using dialyzers of different surface areas, varying blood and dialysate flow rates, varying times of each treatment, or perhaps using more frequent treatments. The ideal treatment for renal failure would mimic the human kidney and be both efficient and continuous.

Conventional HD is efficient but intermittent. By contrast, peritoneal dialysis (PD) is continuous but inefficient. While peritoneal treatments are often better tolerated than those of HD with slow continuous fluid and solute removal, they often do not provide adequate dialysis, especially for larger patients. Whatever manip- ulations that exist for improving the efficiency of PD, they are ultimately limited by the surface area of the peritoneal membrane and the volume that it can contain. In contrast, $\mathrm{HD}$ can easily be improved by decreasing its intermittence. Although not new in concept, there is currently growing interest in daily HD.

\section{Rational for Daily HD}

The rationale for daily HD is based on the fact that 2 hours of dialysis performed only six times a week (total 12 hours), in theory, will significantly increase the weekly removal of urea as compared to the corresponding standard treatment regime of 4 hours three times a week. Most of the urea removed (approximately 60\%) during a 4-hour dialysis takes place during the first half (2). Therefore limiting the session to the first 2 hours will decrease the removal capability by approximately $40 \%$ each treatment, but it will produce a significant increase when done on a weekly basis. Simple mathematics indicate this to be by $20-40 \%$ (depending on 6 or 7 days of treatment) for a standard efficiency dialyzer (urea clearances of $150-200 \mathrm{ml} / \mathrm{min}$ ).

The gain in terms of absolute global weekly removal with short daily HD with respect to standard HD increases progressively with the increase in the clearance (efficiency) because it will increase the percentage removal during the first 2 hours. Percentage removals of up to $75 \%$ with a very high-efficiency dialyzer can be attained (2), and again simple math indicates that the global weekly removal with six times a week dialysis will exceed that of standard HD by $50 \%$. The gain by using high-efficiency dialyzers in daily HD is indeed better than with standard HD. With the latter, for example, increasing the dialyzer clearance from 150 to $200 \mathrm{ml} / \mathrm{min}$ (a 33\% increase of clearance) will be associated with an approximate increase of $23 \%$ in weekly waste removal; an equivalent $33 \%$ increment in clearance from 200 to $267 \mathrm{ml} / \mathrm{min}$ will only lead to a $16 \%$ increase in waste removal (2). It is logical to conclude that it is advantageous to use high clearances coupled with an increase in frequency despite the reduction in the length of each dialysis session.

In actual clinical practice the effect of this favorable situation is blunted by the progressive decrease in predialysis blood solute levels during the first 2-3 weeks of treatment, until a new steady state is reached. Thus urea extraction will decline and weekly urea removal will decrease until a new equilibrium is established. This will be at a level that is more advantageous for the patients since the predialysis concentrations of solute, for example, urea, creatinine, potassium, hydrogen ion, are reduced throughout the week, as are the time-averaged concentrations of these solutes and, perhaps of even more importance, the time-averaged deviations in their concentrations. This is particularly true with hydrogen ion and thus leads to greatly improved control of acid-base equilibrium, with blood levels of $\mathrm{pH}$ and bicarbonate remaining very steady and within the normal range. As intradialytic water removal is also easily performed without 
evidence of cardiovascular instability, the whole "unphysiology" of standard HD (3) is improved.

Although there is currently great interest in daily HD, the idea is anything but new. The first description of daily dialysis was in 1969 when DePalma et al. (4) reported on seven patients that had begun dialysis five times a week for 4-5 hours per session the previous year. The reasons for changing to more frequent dialysis included severe intradialytic hypotension and severe interdialytic hypertension. Both of these (and other medical problems for which daily dialysis was used) were solved and all patients experienced better appetite, weight gain, and better biochemistries in spite of dietary restrictions being lifted. DePalma et al.'s (4) daily dialysis program continued into the early 1970 s but was abandoned due to a combination of reimbursement and technical problems.

Between 1975 and 1981, Snyder et al. (5), Louis et al. (6), and Manohar et al. (7) reported on a total of 10 patients dialyzed daily for periods ranging from 2 months to 7.5 years. These patients, previously dialyzed three times a week for $15 \mathrm{hr} / \mathrm{wk}$, were switched to 2 hours of dialysis five times a week. All observations made on these patients were favorable, yet the program was abandoned in the mid 1980s again because of the U.S. dialysis reimbursement schedule.

In 1979 Bonomini et al. (8) reported on six patients dialyzed 6-12 months for 3-4 hours 5 days a week. These patients had been placed on daily dialysis for a variety of medical reasons, as well as severe subjective complaints, all of which improved.

Since 1980, a slow but now accelerating growth in investigators examining the concept of daily HD has been led by Buonochristiani in Italy, Pierratos in Canada, and Ting and Lockridge in the United States. The largest number of patients with the longest observations of daily dialysis has come from Buonochristiani et al. Starting in 1982, they have now treated close to 100 patients with an ongoing program.

A radically different approach to daily dialysis has been taken by the Toronto group first led by the late Robert Uldall and lately by Andreas Pierratos. The Toronto program started in 1994 and they have used, almost exclusively, long, slow overnight HD at home with the patient dialyzing 5-7 nights a week. They have reported on the excellent well being of their patients (9).

Ting et al. (10) used brief 1.5- to 2-hour daily dialysis as a form of rescue therapy, treating a number of patients with serious medical problems and dialysis intolerance. They are treated six times a week in-center with conventional dialysis equipment and have shown considerable benefit, with in many cases, resolution of the medical problems necessitating that form of therapy and an improvement in quality of life. Additional reports have come from Belgium, the Netherlands, France, and other European centers as well as a number in North America. Indeed, in North America there are currently 300 or more patients receiving mainly short daily HD with some receiving long slow nocturnal dialysis (Lockridge $\mathrm{R}$, personal communication). A detailed review of the literature is given by Kjellstrand and Ing (11) which expands on the individual studies mentioned.
The London, Ontario Daily/Nocturnal HD Study

Despite the interest in daily HD, there are as yet no randomized prospective studies comparing "hard" outcome data in patients dialyzed conventionally with either the short daily or long slow nocturnal dialysis methodologies. The London Study, the first to obtain comparative data in this field, commenced in November 1998 and was completed by the end of 2001. It was a 3year observational study designed to compare 10 patients receiving short daily HD to 10 patients receiving long slow nocturnal dialysis. All patients were to be observed for at least 18 months and the treatments were provided in the home environment. The choice of an observational study was made deliberately because of the anticipated impact of patient preferences regarding the assigned arm of the experimental treatments. Thus randomization would be fraught with difficulties and certainly would influence patients' quality of life. All patients were studied in detail while still on conventional HD and then serially while on the experimental daily/nocturnal program. For each patient selected for the study, a cohort control was obtained matched as closely as possible for age, sex, comorbidity, and the original dialysis modality (in-center HD, satellite HD, self-care HD, home HD, or peritoneal dialysis) and from the center in which that patient was previously treated.

This investigation is now complete and its results have been presented to the Ontario Ministry of Health (the funding agency for the study). The study results, in preparation for publication as a special supplement to the American Journal of Kidney Diseases, show that quotidian dialysis as compared with conventional thrice-weekly HD provides improvement in a number of physiologic areas without any evidence of harm. It is associated with very significant improvement in the patients' perception of their quality of life. The economic analysis of these home-based therapies is entirely favorable and will be seen as financially attractive to the Ministry of Health as well as other providing agencies. The study group has therefore recommended to the Ministry of Health that this modality of treatment become a recognized form of therapy and that an appropriate funding mechanism be established. Specific recommendations in this regard have been offered for consideration by the Ministry of Health.

\section{Future of Quotidian HD}

It is the authors' firm conviction that within a year a number of Canadian provincial governments (responsible for the delivery of health care) will create funding mechanisms for this form of therapy. At that point there will be a swing to this form of therapy, certainly in the home setting, where cost-effectiveness is almost guaranteed. The question then becomes what percentage of the total dialysis population will be managed in a home environment. The London, Ontario, study has taken on patients with significant comorbidities who were previ- 
ously being treated in in-center HD units and has proven that they can manage at home. A conservative estimate is that $20 \%$ of the total HD population will be able to perform this therapy in the home environment. This alone will have a huge impact on the health care economics of renal replacement therapy.

This does not imply that daily dialysis will only be a home therapy. Many patients who prefer daily HD may wish to remain in-center for various reasons, such as inability to self-cannulate, vascular access, housing restrictions, and others. High-risk patients with cramps, hypotensive episodes, volume overload, and/or interdialytic hypertension may benefit from five or six times a week, short-hours, high-efficiency dialysis in the in-center setting when it is logistically feasible. This might be done on a permanent basis or on a temporary basis as a form of "rescue therapy."

To accommodate these changes, in-center units will have to alter their mind-sets regarding scheduling and have improved dialysis equipment available for the particular needs of daily dialysis. The development of an "in situ" reuse system of dialyzers and lines will be of benefit not just economically but for patient convenience. The overall improvement in well-being with reductions in morbidity, hospitalizations, and the use of erythropoietin and other medications will, over time, show overall benefit to the end-stage renal disease (ESRD) population.

These changes in dialysis practice are likely to become more widespread in Canada and Europe long before they are seen in the United States due to the peculiar reimbursement issues that many perceive stultify advancement in the United States. The National Institutes of Health (NIH) has shown initiative in recognizing the growing swell of interest in daily dialysis by recently holding a task force meeting. As usual, it is likely that the NIH will want a randomized prospective study to compare at least short-hours daily HD against conventional therapy. Whether nocturnal HD can be studied is doubtful since this is a home HD therapy, an area in which there is currently little experience in the United States.

What would seem to be a reasonable approach would be to set up a randomized prospective study of shorthours daily HD versus three times a week, with each group being assigned the same number of total hours per week. The study should be carried out in-center on patients with high morbidity scores and with high complication rates so that the impact of the experimental treatment on potential improvement is discernible within a relatively short period of time.

\section{Conclusion}

The HEMO study has now shown us that conventional three times a week HD targeting a single-pool
$K t / V$ greater than $1.25 /$ treatment does not influence outcome. Increasing the time of an intermittent therapy is associated with diminishing returns in toxin removal, but could be of benefit in volume control. The only way to truly increase toxin removal is to increase the frequency of the therapy. Even this may not explain the benefits obtained by more frequent therapy. It could be the approach to a more continuous state that provides the benefit.

We cannot be complacent and say that there is no need to seek out these benefits. The mortality rates of patients undergoing HD, especially in North America, attest to that. A change is needed and the time has come to look in detail at daily HD. Reports going back as far as 1969 uniformly indicate benefit as compared to conventional three times a week therapy.

To the protagonists, daily HD is seen as a costeffective form of therapy that is more physiologic than conventional HD, and produces better outcomes and improves the quality of life of dialysis patients. The skeptics do not appear to doubt the theoretical advantages of this form of therapy, but believe that the increased frequency of dialysis treatment will be unacceptable to patients and that major barriers exist relating to bureaucratic and reimbursement issues. Time will show them that once patients are exposed to this form of therapy, they will never wish to return to conventional HD. Bureaucracy will find, as a minimum, that any increased expenditure has been justified by increased well-being and even that, over time, global costs are reduced.

\section{References}

1. Lowrie EG, Laird NM, Parker TF, Sargent JA: Effect of hemodialysis prescription on patient morbidity: report from the National Cooperative Dialysis Study. N Engl J Med 305:1176-1181, 1981

2. Buonocristiani U, Fagugli R, Quintaliani G, Kuluiranu H: Rationale for daily dialysis. Home Hemodial Int 1:12-18, 1997

3. Kjellstrand CM, Evans RL, Peterson RJ, Shideman JR, von Hartitzsch B, Buselmeier TJ: The "unphysiology" of dialysis: a major cause of dialysis side effects? Kidney Int 7:S30-S34, 1975

4. DePalma JR, Pecker EA, Mazwell MHA: New automatic coil dialyzer system for "daily" dialysis. Proc Eur Dial Transplant Assoc 6:26034, 1969

5. Snyder D, Louis BM, Gorfien P, Mordujovich J: Clinical experience with long-term, brief, "daily" hemodialysis. Eur Dial Transplant Assoc 11:128 135,1975

6. Louis B, Patel TG, Pinedo A, Snyder D, Gorfein P: Clinical experience with long-term 5 days-a-week hemodialysis. Proc Dial Transplant Forum 5:58-60, 1975

7. Manohar NL, Louis BM, Gorfien P, Lipner HI: Success of frequent short hemodialysis. Trans Am Soc Artif Intern Organs 27:604-609, 1981

8. Bonomini V, Mioli V, Albertazzi A, Scolari P: Daily-dialysis programme: indications and results. Proc Eur Dial Transplant Assoc 9:44-52, 1972

9. Pierratos A, Ouwendyk M, Francoeur R, et al.: Nocturnal hemodialysis: three year experience. J Am Soc Nephrol 9:859-868, 1998

10. Ting G, Freitas T, Saum N, Vas S, Raj DSC, Ecclestone A, Langos V, Uldall R: Early metabolic, hematological, clinical and life quality changes with daily hemodialysis. Perit Dial Int 18:S78, 1998

11. Kjellstrand CM, Ing T: History and revival of a superior dialysis method. ASAIO J 44:117-122, 1998 


\section{Francesco Locatelli}

Department of Nephrology and Dialysis, A. Manzoni Hospital, Lecco, Italy

During the last meeting of the National Kidney Foundation (NKF) in Chicago, the results of the HEMO study were presented; they were, unfortunately, not as expected. The main objective of the study was to evaluate the effect of the following interventions on mortality and morbidity in four patient groups: high-flux versus lowflux membranes and high versus standard dialysis dose (Table 1). Further objectives included assessment of cardiovascular hospitalization or death, infectious hospitalization or death, nutritional status, comorbidity, access-related problems, and quality of life. Patients had to be on dialysis for $\geq 3$ months and have a serum albumin greater than $26 \mathrm{~g} / \mathrm{L}$. The observation period was up to 5 years, with substitution of dropout patients. Reuse of dialyzers up to 20 times was allowed in the high-flux group. The main characteristics of the enrolled patients at the time of inclusion were mean age 58 years, mean time on dialysis 3.7 years, mean $s p K t / V 1.6$, mean serum albumin $36 \mathrm{~g} / \mathrm{L} ; 60 \%$ of the patients were treated with high-flux membranes.

The dialyzers used during the study for the low-flux group were mainly the CA 210 and F8 and the CT 190 and F80 for the high-flux group. The $K t / V$ and urea reduction rate (URR) values obtained in the standard and high dialysis dose groups, respectively, were as follows: $s p K t / V, 1.32$ and 1.72 ; eqKt/V, 1.16 and 1.53; and URR (\%), 66 and 75 . With regard to the primary outcome, there were 440 deaths in the standard-dose group and 431 in the high-dose group; 442 deaths occurred in the low-flux membrane group and 429 in the high-flux membrane group.

Considering the population as a whole, no effect of higher dialysis dose was observed. In a subanalysis of female patients, better survival was seen in the high- $K t / V$ group (relative risk [RR]: $0.81 ; p=0.02$ ), that is, a $19 \%$ reduction in relative risk of mortality; in males, no significant effect was found. For the patient population as a whole, a slight statistically insignificant reduction in mortality risk was observed in the high-flux group.

A subanalysis of those patients on dialysis for more than 3.7 years revealed better survival in the high-flux group (RR $0.68 ; p=0.001$ ), while for patients on dialysis for less than 3.7 years there was no effect at all. Thus the answers were not as expected to the two questions: 1) Does a dialysis dose above currently accepted minimum levels improve the outcome of patients followed up to 5 years? 2) Do high-flux membranes offer an advantage independent of the dialysis dose?

In my opinion the main limitations of the HEMO study lie in the fact that $60 \%$ of the enrolled patients

Address correspondence to: Francesco Locatelli, MD, Department of Nephrology and Dialysis, A. Manzoni Hospital, Via Dell'Eremo 9/11, 23900 Lecco, Italy, or email: nefrologia@ospedale.lecco.it.

Seminars in Dialysis-Vol 16, No 1 (January-February) 2003 pp. 20-21 were previously treated with high-flux dialysis, and that the mean $s p K t / V$ at the time of inclusion was relatively high (a mean value of $1.6 \pm 0.3$ ). Therefore it is very likely that some of the patients enrolled in the standard dialysis dose group decreased their dose of dialysis, and that patients previously on long high-flux dialysis were enrolled in the low-flux dialysis group. Thus a carryover effect may not be excluded. Moreover, the positive selection of the patients surviving an average of 3.7 years created a group not actually representing the dialysis population. The mean age of the patients at inclusion in the study was also far younger (58 years after an average of 3.7 years on dialysis) than the general end-stage renal disease (ESRD) population (61 years at the start of dialysis); this should also be considered in the interpretation of the data. Other factors influencing the representativeness of the sample were the exclusion of patients with plasma albumin $\$ 6 \mathrm{~g} / \mathrm{L}$, who have a particularly high risk of morbidity and mortality and who could possibly benefit more from intensive dialysis (flux and $K t / V$ ). Due to this positive selection of patients, doubts on the general applicability of these results are emerging.

One of the aims of the protocol was to compare the effect of the dialyzer flux. However, the reuse practice allowed by the protocol (up to 20 times) might have changed the performance characteristics of the dialyzers in a way that the differentiation between high flux and low flux, and consequently their effect on outcome, might become indistinct. It has been shown that CT190 (high flux), once reprocessed with Renalin, decreases $\beta_{2}$-microglobulin $\left(\beta_{2} \mathrm{M}\right)$ clearance from a median of about 42 to $12 \mathrm{ml} / \mathrm{min}$ after 10 reuses (1). This last value is very close to the upper limit of $\beta_{2} \mathrm{M}$ clearance selected for the definition of low-flux dialyzers.

It is well known that the outcome of renal replacement treatment is strongly related to the quality of care of the predialysis period. Considering the long treatment time on dialysis before randomization, it is possible that the results of this clinical trial are only partially related to the randomized treatment itself. The dialysis "history" might well confound the effect of the intervention period, which might be even shorter than the preceding period, thus predialysis and prerandomization medical care could also have had an effect on outcome.

In Europe, the Membrane Permeability Outcome (MPO) study (2) is currently being performed in nine countries and 61 study centers. The main objective of this study is to assess whether mortality is reduced with highflux dialysis as compared to low-flux dialysis. Patients who are on dialysis for no longer than 2 months are randomly allocated to high-flux or low-flux dialyzers. The target patient number is 660 patients who have to be observed for 3-6 years. As of this writing, the number of randomized patients is 589; no substitution of dropout patients is allowed and, according to European dialysis practice, filter reuse is not allowed. 
TABLE 1. Patient groups

\begin{tabular}{lcc}
\hline & Standard double-pool \\
& $K t / V 1.0-1.1$, URR $65 \%$ & High double-pool \\
Low-flux membranes, $\beta_{2} \mathrm{M}$ clearance $<10 \mathrm{ml} / \mathrm{min}$ & $\mathrm{A}$ & $\mathrm{C}-1.5$, URR $75 \%$ \\
High-flux membranes, $\beta_{2} \mathrm{M}$ clearance $>20 \mathrm{ml} / \mathrm{min}$ & $\mathrm{C}$ & $\mathrm{D}$ \\
\hline
\end{tabular}

The main differences between the HEMO and MPO studies are that only new patients without dialysis "history" (incident patients) are included in the MPO trial and only one intervention (membrane flux) is evaluated. Moreover, in the MPO study there is no exclusion of patients with low serum albumin (those who might benefit more from high-flux dialysis), no reuse of dialyzers is allowed, and dialysis prescription is according to European practice (no attempt to minimize treatment length).

The limitations of the HEMO study and the resultant difficulties in interpreting its results underline the importance of the MPO study. The hope is to come to a more definitive conclusion on the effect of high-flux membranes on patient outcomes.

In conclusion, it is undeniable that the HEMO study results are very disappointing, considering that it has been the opinion of most nephrologists that an increase in the dose of HD, and the use of high-flux membranes, could positively affect patient survival. This opinion has been based mainly on the results of observational evidence very recently supported by the preliminary results of Dialysis Outcomes and Practice Patterns Study
(DOPPS). However, some of the noted drawbacks in the HEMO study suggest that the study results should not be considered conclusive. In fact, the general applicability of the information coming from clinical trials may be limited by the characteristics of the studied patients and practice patterns. The differences between the HEMO study (U.S. prevalent patients, substitution of the dropouts, reuse of dialyzers, some exclusion criteria such as low albumin levels) and the MPO study (European incident patients, no substitution of the dropouts, no dialyzer reuse) make the results of the ongoing MPO study very important for clarifying the effect of membrane flux on patient outcomes.

\section{References}

1. Cheung AK, Agodoa LY, Daugirdas JT, Depner TA, Gotch FA, Greene T, Levin NW, Leypoldt JK: Effects of hemodialyzer reuse on clearances of urea and $\beta_{2}$-microglobulin. J Am Soc Nephrol 10:117-127, 1999

2. Locatelli F, Hannedouche T, Jacobson S, La Greca G, Loureiro A, MartinMalo A, Papadimitriou M, Vanholder R: The effect of membrane permeability on ESRD: design a prospective randomized multicentre trial. J Nephrol 12:85-88, 1999 